\title{
El prestigio de las editoriales académicas con libros en Ciencias Sociales y Humanidades en Brasil
}

\section{The prestige of the academic publishers with books in Social Sciences and Humanities in Brazil}

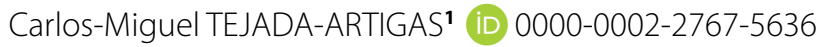 \\ Elea GIMÉNEZ-TOLEDO² (iD 0000-0001-5425-0003 \\ Aline Borges de OLIVEIRA' ${ }^{1}$ (iD) 0000-0001-5877-3290
}

\section{Resumen}

El libro es un importante medio de comunicación científica en Ciencias Sociales y Humanidades, es por ello por lo que se debe potenciar su correcta evaluación. Así, en el presente artículo se exponen las distintas metodologías de valoración de libros académicos que aplican las agencias de Brasil y de España y a continuación se ofrecen resultados de un estudio sobre el prestigio de las editoriales. El cuestionario por el que se han obtenido los datos fue enviado a 16.542 directores de grupos de investigación en Ciencias Sociales y Humanidades y se recibieron 1.977 respuestas (12\%). Los resultados son novedosos, ya que es la primera vez que se realiza un estudio de percepción de la calidad de las editoriales en Brasil, a partir de la opinión de los especialistas. Como ejemplo del trabajo realizado, se presentan las editoriales con mayor prestigio en Educación y, como en el resto de las áreas, hay un núcleo pequeño de editoriales brasileñas que son las que concentran más votos. En el caso de las editoriales extranjeras, el prestigio no está tan concentrado, siendo Portugal, Francia y España los países de procedencia de estas editoriales. Estos resultados pueden servir de forma orientativa en los procesos de evaluación de la actividad científica en Brasil. Además, los editores pueden tener conocimiento de cómo son percibidos y para los autores también es importante conocer esta información cuando tengan que elegir una editorial para presentar sus manuscritos.

Palabras clave: Calidad. Editoriales. Evaluación científica. Publicaciones científicas.

\begin{abstract}
Book are important means of scientific communication in the Social Sciences and Humanities. That is why their correct evaluation should be continuously improved. In this article, we present the different methodologies for the valuation of academic books applied by the Brazilian and Spanish agencies, as well as the results of a study about the prestige of publishers. The

\footnotetext{
1 Universidad Complutense de Madrid, Facultad de Ciencias de la Documentación, Departamento de Biblioteconomía y Documentación. Calle Santísima Trinidad, 37, 28010, Madrid, España. Correspondencia para/Correspondence to: C.-M. TEJADA-ARTIGAS.E-mail:<cmtejada@ucm.es>.

2 Instituto de Filosofía, Centro de Ciencias Humanas y Sociales, Consejo Superior de Investigaciones Científicas. Madrid, España.

Artículo basado en la tesis de A. B. OLIVEIRA, titulada "Los libros en Ciencias Sociales y Humanidades en Brasil: un estudio a partir de los investigadores y de las editoriales". Universidad Complutense, Madrid, 2018.

Apoyo: Ministerio de Economía y Empresa - FEDER (CSO2015-63693-P).

Recibido el 30 de mayo de 2019, presentado el 21 de julio de 2020 y aprobado el 21 de agosto de 2020.
}

Como citar este artículo/How to cite this article

Tejada-Artigas, C.-M.; Giménez-Toledo, E.; Oliveira, A. B. El prestigio de las editoriales académicas con libros en Ciencias Sociales y Humanidades en Brasil. Transinformação, v. 32, e190043, 2020. https://doi.org/10.1590/2318-0889202032e190043 
questionnaire through which the data was obtained was sent to 16,542 directors of research groups in humanities and social sciences, and 1,977 answers (12\%) were obtained. The results are novel, as it is the first time that a study regarding the quality perception of publishers in Brazil is carried out based on the specialists' opinions. The most prestigious publishers in the area of Education are presented and, as in other areas, there is a small nucleus of Brazilian publishers that concentrate the most votes. In the case of foreign publishers, prestige is not so concentrated, with Portugal, France or Spain as the origin countries of these publishers. These results can serve as a guide of scientific evaluation processes in Brazil. In addition, the research might provide editors with knowledge about how they are perceived and help authors choose a publisher to submit their manuscripts.

Keywords: Scientific literature. Publishers. Quality. Scientific evaluation.

\section{Introducción}

El libro es un medio importante en la publicación científica de las Ciencias Sociales y Humanidades (CSH), tal y como muestran distintos estudios realizados a lo largo del tiempo (Broadus, 1971; Hicks, 2004; Nederhof, 2006; JordiArdanuy, 2009; Giménez-Toledo, 2016; Engels et al., 2018; Giménez-Toledo et al., 2019), e investigadores y profesionales de cualesquiera de estas disciplinas eligen el libro como medio de comunicación científica de sus resultados de investigación. Un estudio reciente (Kulczycki et al., 2018) cifra en el 44,8\% las publicaciones de CSH en forma de libro en un conjunto de ocho países europeos. Así pues, al evaluar la producción científica de estos investigadores, es necesario prestar una atención adecuada al libro académico. Para apoyar esos procesos de evaluación se han desarrollado en los últimos años sistemas que valoran la calidad de los libros, mediante una evaluación cualitativa de cada título, como es el caso de Qualis Libros en Brasil o bien mediante métodos indirectos, como puede ser la calidad o el prestigio de las editoriales o las citas que los libros reciben. Como ejemplo de evaluación de editoriales, se pueden citar los listados categorizados de Current Research Information System de Noruega y los rankings de prestigio de las editoriales Scholarly Publishers Indicators (SPI). Por otra parte, Book Citation Index (Clarivate Analytics) y Scopus Book Titles Expansion Program (Elsevier) aportan información sobre citas a libros. Una revisión de los modelos de evaluación existentes y aplicados en Europa puede encontrarse en Giménez-Toledo et al. (2016; 2019).

En el presente artículo se exponen las distintas metodologías de evaluación de libros académicos que aplican las agencias de Brasil y de España y, a continuación, se ofrecen resultados de un estudio sobre el prestigio de las editoriales brasileñas. Así, se muestran los resultados de una consulta realizada a investigadores brasileños sobre el prestigio de las editoriales académicas, con el objetivo de contar con información relevante, objetiva y obtenida con metodología científica que pueda ayudar en los procesos de evaluación de la actividad investigadora en Brasil.

\section{La evaluación de los libros científicos en CSH en Brasil y España}

En Brasil, la Coordenação de Aperfeiçoamento de Pessoal de Nível Superior (CAPES), tiene su origen en el Decreto n 29.741, del 11 de julio de 1951, que fija su objetivo de "asegurar la existencia de personal especializado en cantidad y calidad suficientes para atender las necesidades de los proyectos públicos y privados que tienen como objetivo el desarrollo del país" (Brasil, 1951, art. 2, traducción nuestra)³. Así, es la agencia más importante en Brasil que vela por el desarrollo de los programas de posgrado. Según Zancan (2000), el rápido desarrollo de la investigación en las universidades brasileñas, entre las décadas de 1970 y 1990, se explica por el hecho de que el gobierno federal, en las últimas décadas, financió la apertura de cursos de posgrado con la finalidad de poner en práctica la investigación en las universidades. Leta (2012, p. 51, traducción nuestra) ${ }^{\mathbf{4}}$ confirma que "la ciencia brasileña está completamente en manos de programas de posgrado".

\footnotetext{
${ }^{3}$ Original: "assegurar a existência de pessoal especializado em quantidade e qualidade suficientes para atender às necessidades dos empreendimentos públicos e privados que visam ao desenvolvimento do país" (Brasil, 1951, art. 2).

4Original: "a ciência brasileira está inteiramente nas mãos de programas de pós-graduação" (Leta, 2012, p. 51).
} 
En 1976 comienzan sus trabajos de evaluación de esos programas de posgrado de forma cualitativa, llevados a cabo por expertos que representaban cada área del conocimiento, pero hasta 2009 no se incorporan los criterios para la evaluación de los libros académicos. Esto solo ocurrió después de la aprobación por parte del Consejo Técnico-Científico de la Educación Superior (CTC-ES) del Roteiro para Classificação de Livros (Guion para Clasificación de Libros) (Consejo Técnico-Científico de la Educación Superior, 2009). Esta hoja de ruta para la evaluación de los libros incluye una primera parte en la que se destaca la importancia del libro científico, para a continuación sugerir un modelo de ficha de evaluación. Miranda y Mugnaini atribuyen a este instrumento el que se reconozca al libro como publicación científica y señalan que "puede observarse un aumento de la preocupación por la calidad del contenido de la obra entre los dos últimos trienios [2004-2006 y 2007-2009], denotando que la clasificación de libros tiende a consolidarse en la política científica nacional" (Miranda; Mugnaini, 2014, p. 7, traducción nuestra)5.

El instrumento de evaluación de libros está dividido en tres partes: Parte l: datos de identificación de la obra, que reúne unos datos más bien bibliográficos; Parte ll: evaluación por parte de la comisión de clasificación de libros, en la que se consideran los aspectos formales, como el tipo de obra, de autores y de editoriales; y la Parte III: evaluación del contenido de la obra, que está centrada en la relevancia, la innovación y la potencialidad del impacto de la obra.

Se establecen cinco categorías: L1, L2, L3, L4 y LNC, quedando las dos superiores, L3 y L4, reservadas para las obras de mayor importancia en el desarrollo científico del área y en la formación de doctores y posgraduados. Además, el estrato LNC es para los libros que no logran ser evaluados. Pero este documento es más bien un guion ya que"cada área elaborará su proprio instrumento que contendrá esas tres partes y tendrá este modelo como base, pudiendo añadir o suprimir ítems de evaluación" (Consejo Técnico-Científico de la Educación Superior, 2009, p. 7, traducción nuestra) $)^{6}$.

Son diversos los trabajos que han analizado la evaluación de Capes por áreas concretas: Geografía (Suertegaray, 2013), Lingüística/Letras (Jobim, 2010) y Psicología (Menandro et al., 2011; Costa; Yamamoto, 2015). Además, hay otros dos estudios que se han centrado específicamente en el libro (Miranda; Mugnaini, 2014; Rodrigues et al., 2018). El primero de ellos analiza el contenido de los "Documentos de Área" de las Grandes Áreas de Ciencias Sociales, Humanas y lingüística, Letras y Artes, de los últimos tres trienios de la evaluación de Capes (2010-2012, 2007-2009 y 2004-2006). El segundo trabajo, más reciente, analiza la evaluación de Capes en el cuadrienio 2013-2016 para lo que tienen en cuenta los 49 documentos de área. Aunque el artículo de revista es el tipo de publicación más valorada en 32 áreas $(65,3 \%)$ se valora también el libro. Son 16 (50,0\%) las áreas que tienen un guion para la evaluación de los libros. Uno de los resultados que se puede destacar es el siguiente:

Se constata que el libro a pesar de constituirse como una opción de canal de comunicación en más del 50\% de las áreas, se encuentra en posición de desventaja en el Sistema de Evaluación del CAPES, en comparación con el artículo en periódico científico (Rodrigues et al., 2018, p. 20, traducción nuestra).

Como causas de esta insuficiente valoración del libro, los autores señalan dos factores: los problemas de visibilidad y de distribución sobre todo del libro impreso, para lo cual proponen mejoras en su inclusión en las bases de datos y en el desarrollo del modelo de acceso abierto, y al factor sociopolítico que prima a la revista (Rodrigues et al., 2018). En efecto, la falta de publicación de libros en abierto y/o su falta de presencia en plataformas internacionales han sido detectadas en un reciente estudio sobre libros de acceso abierto en Iberoamérica (Giménez-Toledo; Córdoba-Restrepo, 2018).

\footnotetext{
${ }^{5}$ Original: "pode-se notar um aumento da preocupação com a qualidade do conteúdo da obra entre os dois últimos triênios [2004-2006 e 2007-2009], denotando que a classificação de livros tende a consolidar-se na política científica nacional" (Miranda; Mugnaini, 2014, p. 7).

6 Original: "cada área elaborará seu próprio instrumento contendo essas três partes e tendo este modelo como base podendo acrescentar ou suprimir itens de avaliação" (Consejo Técnico-Científico de la Educación Superior, 2009, p. 7).

7 Original: "constata-se que o livro apesar de se constituir como uma opção de canal de comunicação em mais de 50\% das áreas, ele encontra-se em posição de desvantagem no Sistema de Avaliação da CAPES, em comparação ao artigo em periódico científico" (Rodrigues et al., 2018, p. 20).
} 
Por último, es conveniente remarcar que Capes"no tiene un registro de editoriales ni realiza evaluación sobre su calidad» la traducción es nuestra" (Coordenação de Aperfeiçoamento de Pessoal de Nível Superior, [201-]) ${ }^{\mathbf{8}}$ y:

Como en el caso de los periódicos, las orientaciones y criterios del guion se establecieron con el objetivo exclusivo de evaluar la producción intelectual de los programas de porgrado y, por lo tanto, son inadecuadas para evaluaciones individuales de profesores, investigadores y alumnos (Coordenação de Aperfeiçoamento de Pessoal de Nível Superior, 2018) ${ }^{\text {. }}$.

En España, el título V de la Ley Orgánica de Universidades estableció las bases jurídicas para la creación de la Agencia Nacional de Evaluación de la Calidad y Acreditación (ANECA) que en un primer momento fue una fundación adscrita al Ministerio de Educación. La Ley 15/2014, del 16 de septiembre, de racionalización del Sector Público y otras medidas de reforma administrativa, le dotó de su estatuto definitivo como Organismo Autónomo de la Administración Central del Estado. Tiene como objetivo:

La evaluación de la calidad de la educación superior de alcance nacional encargada de realizar actividades de evaluación, certificación y acreditación, del sistema universitario español con el fin de su mejora continua y adaptación al Espacio Europeo de Educación Superior (EEES) (Agencia ..., 2018).

Esta agencia está encargada de la evaluación de la actividad docente e investigadora a nivel institucional (Programa ACADEMIA), la evaluación del profesorado para su contratación (Programa de Evaluación del Profesorado para la contratación) y también de la evaluación de la actividad investigadora a nivel individual (Comisión Nacional Evaluadora de la Actividad Investigadora, CNEAI) para otorgar tramos de investigación, que representan un complemento retributivo para los investigadores que son evaluados positivamente.

Tanto el programa CNEAI como el ACADEMIA tienen en cuenta el sistema de indicadores para editoriales académicas SPI (Scholarly Publishers Indicators) y el sello de Calidad en Edición Académica (CEA-APQ, Academic Publishing Quality). Scholarly Publishers Indicators fue creado en 2011 por el Grupo de Investigación sobre el Libro Académico del Consejo Superior de Investigaciones Científicas. Agrupa:

\footnotetext{
Tres tipos de indicadores para editoriales académicas: prestigio editorial a partir de la opinión de los investigadores; especialización temática según la clasificación de libros en DILVE (Distribuidor de información del libro español en venta) y procesos de selección de originales a partir de la información declarada por las propias editoriales mediante encuestas (Giménez-Toledo; Mañana-Rodríguez; Tejada-Artigas, 2015).
}

La ANECA tiene en consideración los rankings de prestigio de las editoriales españolas y extranjeras que ofrece el sistema (Grupo ..., 2018), tanto el general para el conjunto de disciplinas, como los específicos de cada disciplina, así como los sistemas de selección de originales declarados por las editoriales. Los rankings de prestigio se elaboran a partir de un cuestionario que se remite a los académicos españoles en el que deben señalar las 10 editoriales españolas y extranjeras más prestigiosas en su campo. Esta encuesta ya se ha realizado en tres ocasiones: en el 2012, 2014 y 2018. En la última consulta, además, se incorporaron algunas novedades como la diferenciación de las valoraciones de editoriales en función de si el académico es o no autor de una determinada editorial, así como la identificación de las colecciones más prestigiosas (Giménez-Toledo, 2018).

Por otra parte, el sello de calidad CEA-APQ lo promueve la Unión de Editoriales Universitarias Españolas y cuenta con el aval de la Agencia Nacional de Evaluación de la Calidad y Acreditación, ANECA, y la Fundación Española para la Ciencia y la Tecnología. Su objetivo es:

Reconocer las mejores prácticas dentro de la edición universitaria española y convertirse en un signo distintivo que tanto las agencias de evaluación de la actividad investigadora como la comunidad académica e investigadora podrán identificar fácilmente. Pretende igualmente ser una vía para promover y estimular la calidad en la edición académica (Unión ...., 2018).

\footnotetext{
${ }^{\mathbf{8}}$ Original: "não possui cadastro de editoras nem faz avaliação sobre sua qualidade" (Coordenação de Aperfeiçoamento de Pessoal de Nível Superior, [201-]).

'Original: como no caso de periódicos as orientações e critérios do roteiro foram estabelecidos visando exclusivamente à avaliação da produção intelectual dos programas de pós-graduação e, portanto, são inadequadas para avaliações individuais de professores, pesquisadores e alunos (Coordenação de Aperfeiçoamento de Pessoal de Nível Superior, 2018).
} 
A este sello concurren las colecciones científicas de las editoriales universitarias y se han realizado tres convocatorias hasta el momento, desde 2017, aunque la última está en pleno proceso de evaluación. La Comisión Nacional Evaluadora de la Actividad Investigadora en la actualidad es un programa de la ANECA, pero anteriormente fue un organismo que se creó en 1989 dentro del marco de la Ley de Reforma Universitaria de 1983 y supuso la primera iniciativa concreta de valorar la producción científica del profesorado universitario y de los investigadores del Consejo Superior de Investigaciones Científicas (Cabezas-Clavijo; Torres-Salinas, 2014). Sus estímulos a la investigación han conllevado un incremento en la producción científica y sus efectos son palpables desde los años 90 (JiménezContreras; Moya-Anegón; Delgado-López-Cozar, 2003).

Con la Ley 15/2014, del 16 de septiembre, de racionalización del sector público y otras medidas de reforma administrativa, se modifica la Ley Orgánica 6/2001, del 21 de diciembre, de Universidades, la ANECA asume las funciones de evaluación que llevaba a cabo la CNEAl, convirtiéndose este organismo en un programa de esta agencia nacional. El objetivo del programa CNEAl es evaluar el mérito científico individual para reconocer un complemento de productividad. Este complemento, llamado coloquialmente sexenio porque se puede solicitar cada seis años, conlleva una mejora en el salario. Pero, además, la posesión o no de sexenios lleva consigo una serie de consecuencias en la carrera profesional. Así, tener un sexenio es una condición impuesta por algunas universidades para poder dirigir tesis doctorales o participar en doctorados de calidad. Además, el Real Decreto-ley 14/2012 deja la puerta abierta para que las universidades incrementen la carga docente de aquellos docentes que no cuenten con un tramo de investigación (sexenio) activo.

Pueden concurrir a los sexenios, de forma voluntaria, los funcionarios de carrera de los cuerpos docentes universitarios y de las escalas de Profesores de Investigación, Investigadores Científicos y Científicos Titulares del Consejo Superior de Investigaciones Científicas, presentando sus cinco publicaciones que consideren más relevantes en un periodo de seis años. La evaluación de estas aportaciones se lleva a cabo mediante unos criterios por campos científicos que pueden variar en cada convocatoria. Se atiende sobre todo al medio de difusión empleado, aceptándose como indicio de calidad la publicación en revistas y editoriales de reconocido prestigio. Para el caso de las monografías, se indica de forma general, que:

Se valorarán las editoriales que ocupen posiciones destacadas, en su correspondiente especialidad, en Scholarly Publishers Indicators (SPI) o, en su caso, en otros de características similares. También podrán considerarse, a juicio de la comisión, publicaciones en colecciones de editoriales universitarias que cuenten con el sello de Calidad en Edición Académica (CEA-APQ) (Comisión ..., 2018. p. 115222).

De forma más concreta, son cinco campos, todos ellos de CSH, los que recogen la posibilidad de que los libros sean aportaciones que reflejen la actividad investigadora: Campo 7: Ciencias Sociales, Políticas, del Comportamiento y de la Educación; Campo 8: Ciencias Económicas y Empresariales; Campo 9: Derecho y Jurisprudencia; Campo 10: Historia, Geografía y Artes; y Campo 11: Filosofía, Filología y Lingüística.

La importancia del libro frente al artículo de revista es muy desigual, aunque sobre todo se prima este último, llegando por ejemplo al extremo del Campo 7, Ciencias Sociales, Políticas, del Comportamiento y de la Educación, en el que se exige que para las áreas de Psicología y Biblioteconomía y Documentación de las cinco aportaciones por lo menos cuatro deben ser artículos de revista.

En cuanto a la aplicación del SPI, los criterios de los diferentes campos que tienen en cuenta los libros son también muy dispares. Así, dos campos, Campo 7, Ciencias Sociales, Políticas, del Comportamiento y de la Educación, y el Campo 8, Ciencias Económicas y Empresariales, valoran solo "las que se sitúen en su primer cuartil para las editoriales españolas o en el primero y el segundo para las extranjeras" (Comisión ..., 2018, p. 115209). Otros dos campos, Campo 10, Historia, Geografía y Artes y Campo; 11, Filosofía, Filología y Lingüística, indican que hay que tener en cuenta el ranking de SPI. Por último, el Campo 9, Derecho y Jurisprudencia, su aplicación es más genérica "respecto a libros se considerarán especialmente los publicados en editoriales de reconocido prestigio y con un procedimiento selectivo para la aceptación de originales (según sistemas recogidos en el Scholarly Publishers Indicators)" (Comisión ..., 2018, p. 115213). 


\section{El prestigio editorial como método de evaluación}

Utilizar métodos de evaluación adecuados para las CSH no solo ayuda a mejorar el reconocimiento de la investigación de estas áreas, sino que también contribuye a que la sociedad comprenda la importancia de las contribuciones de las CSH para la solución de los principales desafíos sociales (Ochsner; Hug; Galeron, 2017).

Para que se reconozca al libro académico como publicación científica destacada en las CSH es necesario que las agencias de evaluación o financiación dispongan de indicadores objetivos y transparentes para valorarlo. El documento base para la evaluación de los libros de Capes deja patente esa necesidad señalando que:

"[...] no hay, hasta el presente [2009] bases bibliométricas e indexadores consensuales equivalentes a los adoptados para los periódicos. [...] evaluar la producción intelectual de los programas difundida por medio de libros requiere el desarrollo de criterios propios y de nuevos instrumentos" (Consejo Técnico-Científico de la Educación Superior, 2009, p. 1, traducción nuestra) ${ }^{\mathbf{1 0}}$.

El informe The Metric Tide (Wilsdon et al., 2015) señala algunas características que deben de tener esos sistemas de indicadores tales como que sean públicos, transparentes y que estén elaborados por expertos con la participación de la comunidad científica y editorial.

Además, dado el volumen de la producción científica, los criterios de evaluación deben ser operativos. Aunque la evaluación más idónea de una publicación científica sería la resultante de su lectura - esa es la reivindicación habitual de humanistas y científicos sociales -, es prácticamente imposible abordar esta tarea por cuestiones de tiempo y recursos. Además, los filtros establecidos en las editoriales ya implican una lectura especializada de la obra que permite determinar si el libro es interesante y tiene calidad suficiente para ser publicado o no. Las prácticas editoriales de selección de originales se convierten así en una "marca" de la editorial o de la revista. Sin una buena selección y sin un buen trabajo editorial, un sello no podría contar con un buen catálogo de títulos. Por eso, al considerar el "prestigio" de las editoriales se está valorando su buen hacer en la creación de un catálogo. Desde luego que una editorial puede publicar títulos de calidad desigual, pero, en términos generales, un catálogo tiene una cierta impronta de calidad de la editorial. Además, como siempre en evaluación científica, los indicadores - en este caso sobre editoriales -, deben ser un apoyo para la toma de decisiones, no algo totalmente determinante.

La evaluación de editoriales es algo complejo, pues son muchas las variables que pueden analizarse en torno a una editorial. Generalmente, en lo que concierne al prestigio de las editoriales, se recurre a la opinión de los expertos. Bien mediante paneles de expertos, bien mediante consultas a la comunidad académica correspondiente pueden determinarse los núcleos de editoriales académicas más relevantes (Giménez-Toledo et al., 2016; 2019). La revisión de las metodologías a nivel internacional permite concluir que la intervención de los expertos en la determinación del prestigio no solo es un procedimiento habitual, sino que, además, puede ser más aceptado que otros métodos, debido a que es la propia comunidad académica quien establece ese reconocimiento a las editoriales. Al fin y al cabo, los académicos no solo son lectores especializados de los fondos de una editorial, sino que también son autores y pueden juzgar las experiencias con los sellos editoriales.

\section{Procedimientos Metodológicos}

Este trabajo tiene su precedente en las investigaciones realizadas para la elaboración de Scholarly Publishers Indicators (Giménez-Toledo; Mañana-Rodríguez;Tejada-Artigas, 2015) y, aunque se ha utilizado la misma metodología, la consulta ha permitido tener conocimiento nuevo sobre el sector editorial académico en Brasil y sobre la reputación de los distintos sellos.

\footnotetext{
${ }^{10}$ Original:"[...] não há, até o presente [2009] bases bibliométricas e indexadores consensuais equivalentes aos adotados para os periódicos. [...] avaliar a produção intelectual dos programas veiculada por meio de livros requer o desenvolvimento de critérios próprios e de novos instrumentos" (Consejo Técnico-Científico de la Educación Superior, 2009, p. 1).
} 
Los resultados que se muestran en este artículo se corresponden con una parte del cuestionario, que tuvo como objetivo adicional estudiar los hábitos de publicación de los investigadores brasileños de las CSH (Oliveira, 2018). En lo que concierne al prestigio de las editoriales percibido por los investigadores brasileños, es necesario señalar algunas cuestiones. Aunque la consulta se realizó a investigadores de todas las áreas de Humanidades y Ciencias Sociales, en este trabajo se presentan solo los resultados del campo de la Educación, que obtuvo la mayor tasa de respuesta (24,5\%). Sin embargo, el resto de los resultados será publicado en el sitio web SPI (Grupo de Investigación sobre el Libro Académico, 2018). Por otra parte, este trabajo analiza las preguntas en las que los académicos debían señalar a su juicio, por orden de importancia, las cinco editoriales brasileñas y las cinco extranjeras con más prestigio en su área de conocimiento.

Los destinatarios de la consulta fueron los coordinadores brasileños de los grupos de investigación de las CSH. Para ello, se utilizó el listado de coordinadores del Diretório dos Grupos de Pesquisa no Brasil (Directorio de los Grupos de Investigación en Brasil) del Conselho Nacional de Desenvolvimento Científico e Tecnológico (CNPq) (Conselho Nacional de Desenvolvimento Científico e Tecnológico, [201-]). Se identificaron 19.746 directores de grupos de investigación en CSH y sus respectivos correos electrónicos; entre ellos 2.198 estaban duplicados ya que un mismo académico puede dirigir varios grupos de investigación. Además, se detectaron 1006 direcciones de correo obsoletas, por lo que finalmente la población total fue de 16.542 investigadores en CSH.

Entre los meses de septiembre y diciembre de 2017, se envió un mensaje de correo electrónico en el que se presentaba la investigación y se invitaba a contestar el cuestionario en línea. La tasa de respuesta fue del 12\%, un porcentaje mucho más bajo que el de las consultas realizadas en España (Giménez-Toledo; Mañana-Rodríguez; TejadaArtigas, 2015), pero con un volumen de información valioso, pues se obtuvieron 1.977 respuestas de investigadores de todas las áreas de las CSH de Brasil.

Para procesar la información y poder ofrecer un listado ordenado de editoriales, se aplicó el Indicador de Calidad de Editoriales según los Expertos (ICEE), que considera no solo el número total de menciones a cada editorial, sino también la posición en la que se han mencionado. La formulación del indicador es la siguiente:

$$
\text { ICEE }=\sum_{i=1}^{i=10} \mathrm{ni} \frac{\mathrm{Ni}}{\mathrm{Nj}}
$$

Donde: $\mathrm{Ni}$ = Número de votos recibidos por la editorial en la posición i (de 1 a 10).

$\mathrm{N}_{\mathrm{j}}=$ Número de votos recibidos por todas las editoriales en todas las posiciones.

$\frac{N i}{N j}=W j$

$W_{j}=$ Peso asignado al número de votos recibidos por cada editorial.

En todos los casos se cumple la condición $W_{1}>W_{2}>W_{K} \ldots>W_{10}$. Esta condición es necesaria para garantizar que los pesos asignados a cada posición se corresponden con su sentido (mayor peso asignado a los votos otorgados a las editoriales más prestigiosas). Este sistema de asignación de pesos a las puntuaciones otorgadas depende de la propia distribución de votaciones y, por lo tanto, excluye la posible arbitrariedad en la asignación de los mismos por parte de los investigadores (Giménez-Toledo; Mañana-Rodríguez; Tejada-Artigas, 2015, p. 857).

\section{Resultados}

\section{Distribución de respuestas por áreas y núcleos de editoriales}

Tal como ya se ha señalado, los investigadores identificaban en dos preguntas del cuestionario las cinco editoriales brasileñas y las cinco extranjeras que, a su juicio, cuentan con más prestigio en su área. Como estos académicos podían señalar su pertenencia a varias áreas, sus valoraciones se han tenido en cuenta en cada una de esas áreas, por lo que la suma total de estas valoraciones (2834) es mayor que la de participantes (1977) (Tabla 1). 
Tabla 1. Valoraciones realizadas por áreas y número de editoriales brasileñas y extranjeras señaladas.

\begin{tabular}{|c|c|c|c|c|}
\hline Áreas Capes & $\begin{array}{l}\text { Valoraciones } \\
\text { de editoriales }\end{array}$ & $\begin{array}{c}\text { Porcentaje } \\
\text { sobre el total de } \\
\text { valoraciones (2834) }\end{array}$ & $\begin{array}{c}\text { Editoriales } \\
\text { brasileñas } \\
\text { identificadas }\end{array}$ & $\begin{array}{r}\text { Editoriales } \\
\text { extranjeras } \\
\text { identificadas }\end{array}$ \\
\hline Antropología/Arqueología & 96 & 3,3 & 103 & 107 \\
\hline Ciencia Política y Relaciones Internacionales & 108 & 3,8 & 98 & 95 \\
\hline Educación & 490 & 17,2 & 247 & 267 \\
\hline Filosofía & 134 & 4,7 & 120 & 154 \\
\hline Geografía & 112 & 3,9 & 93 & 91 \\
\hline Historia & 185 & 6,5 & 139 & 207 \\
\hline Psicología & 157 & 5,5 & 129 & 125 \\
\hline Sociología & 186 & 6,5 & 144 & 146 \\
\hline Teología & 43 & 1,5 & 60 & 63 \\
\hline Administración Pública y de Empresas, Ciencias Contables y Turismo & 240 & 8,4 & 123 & 102 \\
\hline Arquitectura, Urbanismo y Diseño & 95 & 3,3 & 91 & 102 \\
\hline Comunicación e Información & 187 & 6,5 & 136 & 146 \\
\hline Derecho & 181 & 6,3 & 103 & 111 \\
\hline Economía & 76 & 2,6 & 69 & 62 \\
\hline Planificación Urbana y Regional / Demografía & 83 & 2,9 & 36 & 75 \\
\hline Servicio Social & 45 & 1,5 & 53 & 37 \\
\hline Artes/Música & 129 & 4,5 & 110 & 160 \\
\hline Letras/Lingüística & 287 & 10,1 & 162 & 222 \\
\hline Total & 2834 & 100,0 & 444 & 732 \\
\hline
\end{tabular}

Fuente: adaptado de Oliveira (2018).

Nota: Capes: Coordenação de Aperfeiçoamento de Pessoal de Nível Superior.

Como se desprende de la Tabla 1, las áreas en las que se ha registrado mayor participación son Educación, Administración Pública y de Empresas y Letras y Lingüística. En consonancia con estudios previos (Giménez-Toledo; Mañana-Rodríguez; Tejada-Artigas, 2015; Giménez-Toledo, 2018), se observa que el número de editoriales extranjeras citadas (732) es mayor que el de las nacionales (444), lo que resulta lógico porque entre las extranjeras se pueden citar editoriales de todos los países del mundo. Sin embargo, no es así en todas las áreas, puesto que en seis de ellas se han señalado más editoriales locales: Ciencia Política y Relaciones Internacionales, Geografía, Psicología, Administración Pública y de Empresas, Ciencias Contables y Turismo, Economía y Servicio Social.

\section{Las editoriales brasileñas con más prestigio: el caso del área de Educación}

Educación fue el área con mayor tasa de respuesta (Tabla 1), con un total de 490 valoraciones. Entre ellas se identifican 247 editoriales brasileñas y 267 extranjeras identificadas, cifras que muestran un similar conocimiento y reconocimiento de las editoriales nacionales y foráneas. En el sector editorial, el campo de la Educación destaca internacionalmente por sellos tan importantes como Pearson Education o McGraw Hill, pero también por la diversidad de editoriales a nivel nacional e internacional. Analizando el número de votos que ha tenido cada editorial hay que destacar que en las editoriales brasileñas se produce una concentración del voto en una serie de editoriales. Así, hay 32 editoriales (12,9\%) que tienen 10 o más votos con editoriales. Entre ellas, hay dos que superan los 100 votos: Cortez y Vozes. En el caso de las extranjeras, tan solo hay 19 (7,1\%) que tienen 10 o más votos y la editorial que más votos ha tenido ha sido Pearson, con 47, frente a la más valorada brasileña, Cortez, con 137. En las dos tablas siguientes se recogen los resultados obtenidos, indicando aquellas editoriales que por lo menos han tenido un ICEE de 10 (Tablas 2 y 3 ). 
Tabla 2. Listado de editoriales brasileñas ordenadas por ICEE.

\begin{tabular}{|c|c|c|}
\hline Editorial & Votos & ICEE \\
\hline Cortez & 137 & 518 \\
\hline Vozes & 127 & 420 \\
\hline Artmed (Grupo A) & 98 & 350 \\
\hline Autêntica & 80 & 243 \\
\hline Papirus & 71 & 222 \\
\hline Autores Associados & 57 & 200 \\
\hline Fundação Editora da Universidade Estadual Paulista (UNESP) & 53 & 179 \\
\hline Companhia das Letras & 54 & 171 \\
\hline Editora da Universidade de São Paulo (EDUSP) & 41 & 142 \\
\hline Saraiva & 39 & 140 \\
\hline Editora CRV & 43 & 123 \\
\hline Martins Fontes & 41 & 118 \\
\hline Boitempo & 33 & 116 \\
\hline Contexto & 33 & 113 \\
\hline Zahar & 32 & 92 \\
\hline Mercado de Letras & 27 & 86 \\
\hline Parábola Editorial & 18 & 67 \\
\hline Ática (Grupo Somos Educação) & 22 & 58 \\
\hline Atlas (Grupo GEN) & 13 & 52 \\
\hline Appris & 17 & 50 \\
\hline Artes Médicas (Grupo A) & 14 & 48 \\
\hline Livraria da Física & 12 & 47 \\
\hline Editora da Universidade Federal de Minas Gerais (UFMG) & 15 & 43 \\
\hline Grupo A & 10 & 43 \\
\hline Editora 34 & 12 & 42 \\
\hline Pontes Editores & 10 & 42 \\
\hline Universidade Estadual de Campinas (UNICAMP) & 16 & 42 \\
\hline Paz e Terra (Grupo Record) & 16 & 41 \\
\hline Editora Universidade Regional do Noroeste do Estado do Rio Grande do Sul (UNIJUÍ) & 12 & 39 \\
\hline Perspectiva & 10 & 38 \\
\hline Civilização Brasileira (Grupo Record) & 12 & 34 \\
\hline Moderna & 8 & 31 \\
\hline Edições Loyola & 11 & 28 \\
\hline Pearson Brasil & 7 & 28 \\
\hline Editora da Universidade Federal de Santa Catarina (UFSC) & 7 & 27 \\
\hline Fiocruz & 8 & 24 \\
\hline Revista dos Tribunais & 5 & 23 \\
\hline Expressão Popular & 8 & 22 \\
\hline Bertrand Brasil (Grupo Record) & 5 & 20 \\
\hline Paulus & 6 & 20 \\
\hline Penso (Grupo A) & 6 & 20 \\
\hline Bookman (Grupo A) & 7 & 19 \\
\hline Sulina (Grupo Meridional) & 5 & 19 \\
\hline Cosac Naify & 6 & 18 \\
\hline Fundação Getúlio Vargas & 7 & 18 \\
\hline Paco Editorial & 9 & 18 \\
\hline Summus & 6 & 18 \\
\hline Hucitec & 5 & 17 \\
\hline
\end{tabular}


Tabla 2. Listado de editoriales brasileñas ordenadas por ICEE.

\begin{tabular}{|c|c|c|}
\hline Editorial & Votos & ICEE \\
\hline Mediação & 7 & 17 \\
\hline Serviço Nacional de Aprendizagem Comercial (SENACSP) & 6 & 17 \\
\hline Brasiliense & 5 & 16 \\
\hline Editora da Universidade Federal da Bahia (EDUFBA) & 6 & 16 \\
\hline Elsevier (Brasil) & 6 & 16 \\
\hline Blucher & 4 & 15 \\
\hline Editora DP\&A & 6 & 15 \\
\hline Grupo Editorial Nacional (GEN) & 4 & 15 \\
\hline Cengage Learning Brasil & 4 & 14 \\
\hline Edições Universidade Federal do Ceará (UFC) & 6 & 14 \\
\hline Pedro \& João Editores & 4 & 14 \\
\hline Editores Associados & 4 & 13 \\
\hline Editora da Universidade Federal de Pernambuco (EDUFPE) & 3 & 13 \\
\hline Rocco & 4 & 13 \\
\hline Editora da Universidade Federal do Paraná (UFPR) & 3 & 12 \\
\hline Editora da Universidade Federal de São Carlos (EdUFSCar) & 4 & 12 \\
\hline Escrituras & 4 & 12 \\
\hline Átomo \& Alínea & 6 & 11 \\
\hline Frère Théophane Durand (FTD) & 5 & 11 \\
\hline Livros Técnicos e Cientificos (Grupo GEN) & 5 & 11 \\
\hline Abril & 3 & 10 \\
\hline Briquet de Lemos & 2 & 10 \\
\hline Casa do Psicólogo (Pearson Clinical Brasil) & 3 & 10 \\
\hline Guanabara Koogan (Grupo GEN) & 2 & 10 \\
\hline Juruá & 4 & 10 \\
\hline
\end{tabular}

Fuente: adaptado de Oliveira (2018)

Nota: ICEE: Indicador de Calidad de Editoriales según los Expertos.

Tabla 3. Listado de editoriales extranjeras ordenadas por ICEE.

1 de 2

\begin{tabular}{lcc}
\hline Editorial & Votos & ICEE \\
\hline Pearson & 47 & 183 \\
Porto & 41 & 160 \\
Routledge & 35 & 125 \\
Oxford University Press & 30 & 98 \\
Springer & 26 & 98 \\
Elsevier & 26 & 89 \\
Cambridge University Press & 26 & 85 \\
McGraw-Hill & 24 & 83 \\
Paidós & 20 & 78 \\
Sage Publications & 19 & 74 \\
Edições 70 & 18 & 68 \\
Fondo de Cultura Económica & 17 & 58 \\
Siglo XXI & 13 & 55 \\
Cengage Learning & 12 & 48 \\
Gallimard & 14 & 45 \\
Thompson Educational Publishing & 14 & 45 \\
Fundação Calouste Gulbenkian & 11 & 43 \\
\hline
\end{tabular}


Tabla 3. Listado de editoriales extranjeras ordenadas por ICEE.

\begin{tabular}{|c|c|c|}
\hline Editorial & Votos & ICEE \\
\hline Presses Universitaires de France & 12 & 43 \\
\hline Bertrand & 10 & 38 \\
\hline Almedina & 7 & 31 \\
\hline Éditions du Seuil & 9 & 31 \\
\hline Palgrave Macmillan & 7 & 31 \\
\hline Consejo Latinoamericano de Ciencias Sociales (CLACSO) & 7 & 28 \\
\hline Harvard University Press & 8 & 26 \\
\hline L'Harmattan & 7 & 24 \\
\hline Grupo Santillana & 8 & 23 \\
\hline John Wiley \& Sons & 7 & 23 \\
\hline Gedisa & 7 & 21 \\
\hline Taylor \& Francis & 6 & 21 \\
\hline The MIT Press & 6 & 19 \\
\hline Anthropos & 4 & 17 \\
\hline Les Éditions de Minuit & 5 & 17 \\
\hline Grao & 4 & 16 \\
\hline Sense & 5 & 15 \\
\hline Gallaudet University Press & 3 & 14 \\
\hline Suhrkamp Verlag & 4 & 14 \\
\hline Dom Quixote & 4 & 13 \\
\hline Editorial Presença & 4 & 13 \\
\hline Fayard & 3 & 13 \\
\hline Addison-Wesley & 3 & 12 \\
\hline Jossey-Bass (John Wiley \& Sons) & 3 & 12 \\
\hline Penguin Books & 4 & 12 \\
\hline Eudeba & 3 & 11 \\
\hline Octaedro & 4 & 11 \\
\hline Afrontamento & 3 & 10 \\
\hline Anagrama & 3 & 10 \\
\hline Ediciones Akal & 4 & 10 \\
\hline Emerald & 3 & 10 \\
\hline Estampa & 3 & 10 \\
\hline LIT Verlag & 2 & 10 \\
\hline Narcea & 3 & 10 \\
\hline Relógio D’Água & 4 & 10 \\
\hline Science & 2 & 10 \\
\hline Visor & 2 & 10 \\
\hline
\end{tabular}

Fuente: adaptado de Oliveira (2018).

Nota: ICEE: Indicador de Calidad de Editoriales según los Expertos.

\section{Conclusión}

Este trabajo presenta resultados de investigación originales sobre el sector editorial académico en Brasil. Es la primera vez que se realiza un estudio de percepción de la calidad de las editoriales en el país a partir de la opinión de los especialistas. Si bien la tasa de respuesta de este estudio es muy desigual por áreas y, globalmente, puede considerarse baja, lo cierto es que el número total de respuestas es alto y permite que se tome en consideración para conocer mejor el sector académico en Brasil y también para obtener una aproximación sobre el prestigio de las editoriales académicas. Los resultados permiten valorar fundamentalmente dos variables: (1) la variedad y diversidad de 
editoriales reconocidas a nivel nacional e internacional y (2) la concentración del prestigio en un núcleo relativamente pequeño de editoriales. Además, con respecto al primer punto señalado, hay que indicar que esta investigación ha permitido crear un directorio de editoriales académicas brasileñas que no existía.

Se puede afirmar que el sector editorial académico en Brasil es amplio, pues llegan a citarse 247 editoriales solo en el campo de la Educación. Editoriales que por un lado tienen carácter público, mientras que por otro son privadas/ comerciales, lo que aporta información sobre los subsectores editoriales y permitirá establecer comparaciones con otros países de la región. Otra clara conclusión es que el prestigio se concentra en unas pocas editoriales académicas. Como se apuntaba anteriormente, solo dos concentran más de 100 votos y solo 32 tienen más de 10 votos. Esta información puede ser suficientemente significativa. Por otra parte, en el terreno internacional, se observa: (1) que el prestigio no está tan concentrado; (2) que se citan editoriales de distintos países del mundo, con presencia de editoriales portuguesas, francesas o españolas que resulta destacada; y (3) que el conocimiento de las editoriales no siempre es muy preciso, tal y como muestra que Gallimard aparezca citada en el campo de la Educación, cuando es un sello fundamentalmente literario.

Aunque es lógico que los investigadores conozcan mejor el sector editorial de su país, lo cierto es que la información que aporta este tipo de estudios sobre las editoriales extranjeras resulta de un interés extraordinario. A partir de ella, se puede conocer algo más acerca de la transferencia de información científica de unos países a otros, de las influencias culturales, de los países que son referentes científicos para otro país y también - esto es interesante para el sector editorial -, de la manera en que algunas editoriales trascienden las fronteras y llegan a otros países.

Este tipo de resultados pueden llegar a ser de utilidad para los propios académicos cuando selecciona la editorial para publicar; para los editores que podrán ver cómo son percibidos y cómo se sitúan frente a la competencia; para los bibliotecarios en la toma de decisiones en sus adquisiciones y también para los evaluadores de la actividad cientíica. Los resultados obtenidos para Educación y para el resto de las áreas (que serán publicados en la web Scholarly Publishers Indicators) podrían servir de forma orientativa en los procesos de evaluación de la actividad científica en Brasil. Asimismo, podrían ser útiles en otros países, cuando se trate de evaluar la producción científica publicada en estas editoriales. En cualquiera de los casos, resulta fundamental no utilizar estos listados de forma automática en la evaluación científica. Pueden aportar información valiosa, pero debería ir acompañada de algunas otras valoraciones, especialmente en los casos de procesos de evaluación de profesores e investigadores.

\section{Colaboradores}

Todos los autores han contribuido en la revisión de la literatura, en el diseño metodológico, en el análisis de los resultados y en las conclusiones. CM TEJADA-ARTIGAS Y E GIMÉNEZ-TOLEDO han redactado el texto.

\section{Referencias}

Agencia Nacional de Evaluación de la Calidad y Acreditación. Presentacíon. Madrid: Aneca, 2018. Disponible en: http://www. aneca.es/ANECA/Presentacion. Acceso en: 15 marzo 2019.

Brasil. Decreto no 29.741, de 11 de julho de 1951. Institui uma Comissão para promover a Campanha Nacional de Aperfeiçoamento de Pessoal de Nível Superior. Brasília: Presidência da República, 1992. Disponível em: http://www2. camara.leg.br/legin/fed/decret/1950-1959/decreto-2974111-julho-1951-336144-publicacaooriginal-1-pe.html. Acesso em: 11 fev. 2019.

Broadus, R. N. The literature of the social science: a survey of citation studies. International Social Science Journal, n. 23, p. 236-243, 1971. Available from: http://citeseerx.ist.psu.edu/ viewdoc/download?doi=10.1.1.566.119\&rep=rep1\&type=pd. Access on: 15 May 2018.
Cabezas-Clavijo, Á.; Torres-Salinas, D. Los sexenios de investigación. Barcelona: UOC, 2014.

Comisión Nacional Evaluadora de la Actividad Investigadora. Resolución de 14 de noviembre de 2018, de la Comisión Nacional Evaluadora de la Actividad Investigadora, por la que se publican los criterios específicos aprobados para cada uno de los campos de evaluación. Boletín Oficial del Estado, n. 285, p. 115199-115222, 2018. Disponible en: https://www.boe.es/ diario_boe/txt.php?id=BOE-A-2018-16138. Acceso en: 13 jun. 2019.

Conselho Nacional de Desenvolvimento Científico e Tecnológico. Diretório dos Grupos de Pesquisa no Brasil. Brasília: CNPq, [201-]. Disponível em: http://lattes.cnpq.br/web/dgp/home. Acesso em: 15 maio 2018. 
Consejo Técnico-Científico de la Educación Superior. Roteiro para classificação de livros. Brasília: Capes, 2009. Disponível em: http:// capes.gov.br/images/stories/download/diversos/RoteiroLivros. pdf. Acesso em: 13 feb. 2019.

Coordenação de Aperfeiçoamento de Pessoal de Nível Superior. Perguntas Frequentes. Brasília: Capes, [201-] Disponível em: http:// www.capes.gov.br/perguntas-frequentes. Acesso em: 15 mar. 2019.

Cordenação de Aperfeiçoamento de Pessoal de Nível Superior. Classificação da produção intelectual. Brasília: Capes, 2018. Disponível em: http://www.capes.gov.br/avaliacao/ instrumentos-de-apoio/classificacao-da-producao-intelectual. Acesso em: 15 jun. 2018.

Costa, J.; Yamamoto, O. H. Produção científica em Psicologia nos cursos de graduação de psicólogos. Arquivos Brasileiros de Psicologia, v. 67, n. 1, p. 20-36, 2015. Disponível em: http://pepsic.bvsalud.org/scielo.php?script=sci_ arttext\&pid=S1809-52672015000100003. Acesso em: 15 jan. 2019.

Engels, T. C. E. et al. Are book publications disappearing from scholarly communication in the social sciences and humanities? Aslib Journal of Information Management, v. 70, n. 6, p. 592-607, 2018. Doi: https://doi.org/10.1108/AJIM-05-2018-0127.

Giménez-Toledo, E.; Mañana-Rodríguez, J.; Tejada-Artigas, C. M. Scholarly publishers indicators: prestigio, especialización y sistemas de revisión de originales en editoriales académicas. El Profesional de la Información, v. 24, n. 6, p. 855-860, 2015. Doi: https://doi.org/10.3145/epi.2015.nov.18.

Giménez-Toledo, E. Malestar: Ios investigadores ante su evaluación. Madrid: Iberoamericana Vervuert, 2016.

Giménez-Toledo, E. et al. Taking scholarly books into account: current developments in five european countries. Scientometrics, v. 107, n. 2, p. 685-699, 2016. Doi: https://doi.org/10.1007/ s11192-016-1886-5.

Giménez-Toledo, E. Estudio cualitativo de las editoriales académicas: la percepción de la comunidad científica española. Madrid: Federación del Gremio de Editores de España, 2018. Disponible en: http://ilia.cchs.csic.es/SPI/spi-fgee/docs/EAEV3. pdf. Acceso en: 19 marzo 2019.

Giménez-Toledo, E.; Córdoba-Restrepo, J. F. Edición académica y difusión: libro abierto en Iberoamérica. Granada: Editorial Universidad del Rosario, 2018. Doi: https://doi.org/10.12804/ th9789587841671.

Giménez-Toledo, E. et al. Taking scholarly books into account, part II: a comparison of 19 European countries in evaluation and funding. Scientometrics, v. 118, n. 1, p. 233-251, 2019. Doi: https://doi.org/10.1007/s11192-018-2956-7.

Grupo de Investigación sobre el Libro Académico. Resultados: rankings SPI. Madrid: Consejo Superior de Investigaciones Científicas, 2018. Disponível em: http://ilia.cchs.csic.es/SPI/ rankings.html. Acceso en: 19 feb. 2019.

Hicks, D. The four literatures of social science. In: Moed, H. F.; Glänzel, W.; Schmoch, U. (Org.). Handbook of Quantitative Science and Technology Research. Dordrecht: Kluwer Academic Publishers, 2004. p. 473-496.

Jiménez-Contreras, E.; Moya-Anegón, F.; Delgado-López-Cozar, $E$. The evolution of research activity in Spain: the impact of the National Commission for the Evaluation of Research Activity
(CNEAl). Research Policy, v. 32, n. 1, p. 123-142, 2003. Doi: https:// doi.org/10.1016/S0048-7333(02)00008-2.

Jobim, J. L. Qualis e separação de áreas: problemas e perspectivas. Revista Anpoll, v. 1, n. 28, 2010. Doi: https://doi.org/10.18309/ anp.v1i28.170.

Jordi-Ardanuy, B. Els estudis bibliomètrics basats en l'anàlisi de citacions en àrees d'humanitats en el període 1959-2008. Bid: Textos Universitaris de Biblioteconomia i Documentació, n. 22, p. 1-23, 2009. Doi: https://dx.doi.org/10.1344/105.000001462.

Kulczycki, E. et al. Publication patterns in the social sciences and humanities: evidence from eight European countries. Scientometrics, v. 116, n.1 p. 463-486, 2018. Doi: https://doi. org/10.1007/s11192-018-2711-0.

Leta, J. Brazilian growth in the mainstream science: the role of human resources and national journals. Journal of Scientometric Research, v. 1, n. 1, p. 44-52, 2012. Doi: https://doi.org/10.5530/ jscires.2012.1.9.

Menandro, P. R. M. et al. Livros à mão cheia: o livro como veículo de produção acadêmica. Psicologia USP, v. 22, n. 2, p. 367-386, 2011. Disponível em: http://www.scielo.br/pdf/pusp/v22n2/ v22n2a05.pdf. Acesso em: 29 mar. 2019.

Miranda, E. C.; Mugnaini, R. Estudo exploratório dos critérios de classificação de livros nas áreas de Ciências Sociais Aplicadas, Humanas e Linguística, Letras \& Artes. In: Encontro Brasileiro de Bibliometria e Cientometria, 4., 2014, Recife. Anais [...]. Recife: UFPE, 2014.

Nederhof, A. J. Bibliometric monitoring of research performance in the social sciences and the humanities: a Review. Scientometrics, v. 66, n. 1, p. 81-100, 2006. Doi: https://doi. org/10.1007/s11192-006-0007-2.

Ochsner, M.; Hug, S.; Galleron, I. The future of research assessment in the humanities: bottom-up assessment procedures. Palgrave Communications, v. 3, 2017. Doi: https://doi.org/10.1057/ palcomms.2017.20.

Oliveira, A. B. Los libros en Ciencias Sociales y Humanidades en Brasil: un estudio a partir de los investigadores y de las editoriales. 2018. Tesis (Doctorado en Documentación) - Universidad Complutense, Madrid, 2018.

Rodrigues, K. O. et al. O livro no sistema de avaliação da CAPES. SciELO 20 Years Repository, p. 1-23, 2018. Disponível em: http:// repository.scielo20.org/documents/article/view/129. Acesso em: 30 nov. 2018.

Suertegaray, D. M. A. Pós-Graduação: uma experiência de avaliação entre a concorrência e a solidariedade. Revista Contexto eEducação, v. 24, n. 81, p. 113-131, 2013. Disponível em: https:// www.revistas.unijui.edu.br/index.php/contextoeducacao/ article/view/1033. Acesso em: 30 jan. 2019.

Unión de Editoriales Universitarias Españolas. Sello de Calidad en Edición Académica. Madrid: Une, 2018. Disponible em: http:// www.selloceaapq.es/. Acceso en: 15 feb. 2019.

Wilsdon, J. et al. The Metric Tide: report of the independent review of the role of metrics in research assessment and management. Los Angeles: Sage Publications, 2015. Doi: https:// doi.org/10.13140/RG.2.1.4929.1363.

Zancan, G. T. Educação científica: uma prioridade nacional. São Paulo em Perspectiva, v. 14, n. 3, p. 3-7, 2000. Doi: https://doi. org/10.1590/S0102-88392000000300002. 